\title{
Monogenic functions in a finite-dimensional semi-simple commutative algebra
}

\author{
S. A. Plaksa and R. P. Pukhtaievych
}

\begin{abstract}
We obtain a constructive description of monogenic functions taking values in a finite-dimensional semi-simple commutative algebra by means of holomorphic functions of the complex variable. We prove that the mentioned monogenic functions have the Gateaux derivatives of all orders. For monogenic functions we prove also analogues of classical integral theorems of the holomorphic function theory: the Cauchy integral theorems for surface and curvilinear integrals, the Morera theorem and the Cauchy integral formula.
\end{abstract}

Introduction. William Hamilton (1843) constructed an algebra of noncommutative quaternions over the field of real numbers $\mathbb{R}$, and developing the hypercomplex analysis began. C. Segre [1] constructed an algebra of commutative quaternions $\left\{x+i y+j z+k t: i^{2}=j^{2}=-1, i j=k, x, y, z, t \in \mathbb{R}\right\}$ over the field $\mathbb{R}$ that can be considered as a two-dimensional commutative semi-simple algebra of bicomplex numbers $\left\{z_{1}+j z_{2}: j^{2}=-1, z_{1}, z_{2} \in \mathbb{C}\right\}$ over the field of complex numbers $\mathbb{C}$.

A theory of functions of a bicomplex variable was developed in papers of many authors (see, e.g., $[2,3,4,5,6]$ ). In particular, in the papers of F. Ringleb [2] and J. Riley [3], it is proved that any analytic function of a bicomplex variable can be constructed with an use of two holomorphic functions of complex variables. In addition, G. Price [4] considered multicomplex

Key Words: Commutative Banach algebra, monogenic function, Laplace equation, Cauchy integral theorem, Cauchy integral formula, Morera theorem.

2010 Mathematics Subject Classification: Primary 30G35; Secondary 35J05.

Received: December, 2013

Revised: January 2014

Accepted: January 2014 
algebras and proved some analogues of results obtained for analytic functions of a bicomplex variable.

A. K. Bakhtin [7] considered the multidimensional complex space $\mathbb{C}^{n}$ as an algebra isomorphic to the $n$-dimensional commutative semi-simple algebra over the field $\mathbb{C}$. He introduced a vector generalization of the module and the argument of a complex number. Using these notions, for functions given in $\mathbb{C}^{n}$, he established analogues of some results of the theory of mappings of the complex plane.

A relation between spatial potential fields and analytic functions given in commutative algebras was established by P. W. Ketchum [8] who shown that every analytic function $\Phi(\zeta)$ of the variable $\zeta=x e_{1}+y e_{2}+z e_{3}$ satisfies the three-dimensional Laplace equation in the case where the elements $e_{1}, e_{2}, e_{3}$ of a commutative algebra satisfy the condition

$$
e_{1}^{2}+e_{2}^{2}+e_{3}^{2}=0
$$

because

$$
\frac{\partial^{2} \Phi}{\partial x^{2}}+\frac{\partial^{2} \Phi}{\partial y^{2}}+\frac{\partial^{2} \Phi}{\partial z^{2}} \equiv \Phi^{\prime \prime}(\zeta)\left(e_{1}^{2}+e_{2}^{2}+e_{3}^{2}\right)=0
$$

where $\Phi^{\prime \prime}:=\left(\Phi^{\prime}\right)^{\prime}$ and $\Phi^{\prime}(\zeta)$ is defined by the equality $d \Phi=\Phi^{\prime}(\zeta) d \zeta$. An algebra is called harmonic if there exists a harmonic triad $\left\{e_{1}, e_{2}, e_{3}\right\}$ satisfying the equality (1). P.W. Ketchum [8] considered the C. Segre algebra of quaternions [1] as an example of harmonic algebra.

I. P. Mel'nichenko [9] noticed that doubly differentiable in the sense of Gateaux functions form the largest algebra of functions $\Phi$ satisfying identically the equality (2), where $\Phi^{\prime \prime}$ is the Gateaux second derivative of function $\Phi$. He proved that there does not exist a three-dimensional harmonic algebra with unit over the field $\mathbb{R}$, but he found all three-dimensional harmonic algebras over the field $\mathbb{C}$ and constructed all harmonic bases in these algebras (see [10]).

Constructive descriptions of monogenic (i.e. continuous and differentiable in the sense of Gateaux) functions in three-dimensional harmonic algebras by means holomorphic functions of the complex variable are obtained in the papers $[11,12,13]$. Such descriptions make it possible to prove the infinite differentiability in the sense of Gateaux of monogenic functions and integral theorems for these functions that are analogous to classical theorems of the complex analysis (see, e.g., [14]).

In this paper we obtain similar results for monogenic functions given in a commutative finite-dimensional semi-simple algebra over the field of complex numbers and give some examples indicating relations between the mentioned functions and multidimensional Laplace equations. 


\section{A constructive description of monogenic functions in a finite- dimensional semi-simple commutative algebra.}

Let $\mathbb{A}_{n}$ be a $n$-dimensional semi-simple commutative associative Banach algebra over the field of complex numbers $\mathbb{C}$, where $2 \leq n<\infty$, and a basis of $\mathbb{A}_{n}$ be formed by idempotents $I_{1}, I_{2}, \ldots, I_{n}$ satisfying the multiplication table:

$$
I_{k}^{2}=I_{k}, \quad I_{k} I_{p}=0, \quad k, p=1,2, \ldots, n, \quad k \neq p .
$$

The unit of $\mathbb{A}_{n}$ is represented as $1=I_{1}+I_{2}+\cdots+I_{n}$

Let us consider the vectors $e_{1}=1, e_{2}, \ldots, e_{m}$ in $\mathbb{A}_{n}$, where $2 \leq m \leq 2 n$, and these vectors are linearly independent over the field of real numbers $\mathbb{R}$. It means that the equality

$$
\sum_{j=1}^{m} \beta_{j} e_{j}=0, \quad \beta_{j} \in \mathbb{R}
$$

holds if and only if $\beta_{j}=0$ for all $j=1,2, \ldots, m$.

Let $E_{m}:=\left\{\zeta=\sum_{j=1}^{m} x_{j} e_{j}: x_{j} \in \mathbb{R}\right\}$ be the linear span of the vectors $e_{1}, e_{2}, \ldots, e_{m}$ over the field $\mathbb{R}$.

Let $\Omega$ be a domain in $E_{m}$. We say that a continuous function $\Phi: \Omega \rightarrow \mathbb{A}_{n}$ is monogenic in $\Omega$ if $\Phi$ is differentiable in the sense of Gateaux in every point of $\Omega$, i.e. if for every $\zeta \in \Omega$ there exists an element $\Phi^{\prime}(\zeta) \in \mathbb{A}_{n}$ such that

$$
\lim _{\varepsilon \rightarrow 0+0}(\Phi(\zeta+\varepsilon h)-\Phi(\zeta)) \varepsilon^{-1}=h \Phi^{\prime}(\zeta) \quad \forall h \in E_{m} .
$$

$\Phi^{\prime}(\zeta)$ is the Gateaux derivative of the function $\Phi$ in the point $\zeta$.

In turn, if $\Phi^{\prime}$ is a monogenic function in the domain $\Omega$, then we denote the Gateaux derivative of the function $\Phi^{\prime}$ by $\Phi^{\prime \prime}$ and call $\Phi^{\prime \prime}$ by the Gateaux second derivative. Further, in the same way we define the Gateaux $s$-th derivative $\Phi^{(s)}$.

Consider the decomposition of function $\Phi: \Omega \rightarrow \mathbb{A}_{n}$ with respect to the basis $\left\{I_{1}, I_{2}, \ldots, I_{n}\right\}$ :

$$
\Phi(\zeta)=\sum_{k=1}^{n} W_{k}\left(x_{1}, x_{2}, \ldots, x_{m}\right) I_{k} .
$$

In the case where the complex-valued functions $W_{k}$ are $\mathbb{R}$-differentiable, i.e.

$$
W_{k}\left(x_{1}+\triangle x_{1}, x_{2}+\triangle x_{2}, \ldots, x_{m}+\triangle x_{m}\right)-W_{k}\left(x_{1}, x_{2}, \ldots, x_{m}\right)=\sum_{j=1}^{m} \frac{\partial W_{k}}{\partial x_{j}} \Delta x_{j}+
$$




$$
+o\left(\sqrt{\sum_{j=1}^{m}\left(\Delta x_{j}\right)^{2}}\right), \quad \sum_{j=1}^{m}\left(\Delta x_{j}\right)^{2} \rightarrow 0,
$$

the function $\Phi$ is monogenic in the domain $\Omega$ if and only if the following Cauchy - Riemann conditions are satisfied in $\Omega$ :

$$
\frac{\partial \Phi}{\partial x_{2}}=\frac{\partial \Phi}{\partial x_{1}} e_{2}, \quad \frac{\partial \Phi}{\partial x_{3}}=\frac{\partial \Phi}{\partial x_{1}} e_{3}, \quad \ldots, \quad \frac{\partial \Phi}{\partial x_{m}}=\frac{\partial \Phi}{\partial x_{1}} e_{m} .
$$

Consider the decompositions of vectors $e_{1}, e_{2}, \ldots, e_{m}$ with respect to the basis $\left\{I_{1}, I_{2}, \ldots, I_{n}\right\}$ :

$$
e_{1}=\sum_{k=1}^{n} I_{k}, \quad e_{j}=\sum_{k=1}^{n} a_{j k} I_{k}, \quad a_{j k} \in \mathbb{C}, j=2,3, \ldots, m .
$$

Consider the linear continuous functionals $f_{k}: \mathbb{A}_{n} \rightarrow \mathbb{C}, k=1,2, \ldots n$, satisfying the equalities

$$
f_{k}\left(I_{k}\right)=1, \quad f_{k}\left(I_{p}\right)=0, \quad p=1,2, \ldots n, \quad p \neq k .
$$

It follows from (6) that the maximal ideal

$$
\mathfrak{I}_{k}:=\left\{\zeta=\sum_{p=1, p \neq k}^{n} \alpha_{p} I_{p}, \quad \alpha_{p} \in \mathbb{C}\right\}
$$

is the kernel of functional $f_{k}$.

Let $M_{k}:=\left\{\zeta \in E_{m}: f_{k}(\zeta)=0\right\}$ for a fixed $k \in\{1,2, \ldots, n\}$. We say that a domain $\Omega \subset E_{m}$ is convex with respect to the set of directions $M_{k}$ if $\Omega$ contains the segment $\left\{\zeta_{1}+\alpha\left(\zeta_{2}-\zeta_{1}\right): \alpha \in[0,1]\right\}$ for all points $\zeta_{1}, \zeta_{2} \in \Omega$ such that $\zeta_{2}-\zeta_{1} \in M_{k}$.

In what follows, we assume that the equality $f_{k}\left(E_{m}\right)=\mathbb{C}$ holds, where $f_{k}\left(E_{m}\right)$ is the image of $E_{m}$ under the mapping $f_{k}$.

Lemma 1. Let a domain $\Omega \subset E_{m}$ be convex with respect to the set of directions $M_{k}$ for some $k \in\{1,2, \ldots, n\}, f_{k}\left(E_{m}\right)=\mathbb{C}$ and $\Phi: \Omega \rightarrow \mathbb{A}_{n}$ be a monogenic function in $\Omega$. If $\zeta_{1}, \zeta_{2} \in \Omega$ and $\zeta_{2}-\zeta_{1} \in M_{k}$, then

$$
\Phi\left(\zeta_{2}\right)-\Phi\left(\zeta_{1}\right) \in \mathfrak{I}_{k}
$$

Proof. Inasmuch as $f_{k}\left(E_{m}\right)=\mathbb{C}$, then there exists an element $e_{2}^{*} \in E_{m}$ such that $f_{k}\left(e_{2}^{*}\right)=i$. Consider the linear span $E^{*}:=\left\{\zeta=x e_{1}^{*}+y e_{2}^{*}+z e_{3}^{*}\right.$ : $x, y, z \in \mathbb{R}\}$ of the vectors $e_{1}^{*}:=1, e_{2}^{*}, e_{3}^{*}:=\zeta_{2}-\zeta_{1}$ and denote $\Omega^{*}:=\Omega \cap E^{*}$. 
Now, the relations (7) is proved in a such way as Lemma 2.1 [11], in the proof of which one must take $\Omega^{*}, f_{k},\left\{\alpha e_{3}^{*}: \alpha \in \mathbb{R}\right\}$ instead of $\Omega_{\zeta}, f, L$, respectively. Lemma 1 is proved.

Let $D_{k}:=f_{k}(\Omega)$ for $k=1,2, \ldots, n$. Let $A_{k}$ be the linear operator which assigns a holomorphic function $F_{k}: D_{k} \rightarrow \mathbb{C}$ to every monogenic function $\Phi: \Omega \rightarrow \mathbb{A}_{n}$ by the formula

$$
F_{k}\left(\xi_{k}\right)=f_{k}(\Phi(\zeta))
$$

where $\xi_{k}=f_{k}(\zeta)$ and $\zeta \in \Omega$. It follows from Lemma 1 that the value $F_{k}\left(\xi_{k}\right)$ does not depend on a choice of a point $\zeta$ for which $f_{k}(\zeta)=\xi_{k}$.

Similar operators $A$ which map monogenic functions taking values in certain commutative algebras onto holomorphic functions of the complex variable are explicitly constructed in the papers $[10,11]$. Furthermore, principal extensions of holomorphic functions of the complex variable are used there as generalized inverse operators $A^{(-1)}$ satisfying the equality $A A^{(-1)} A=A$. It was also established for every monogenic function $\Phi$ that values of the monogenic function $\Phi-A^{(-1)} A \Phi$ belong to a certain maximal ideal $\mathfrak{I}$ of given algebra. Finally, after describing all monogenic functions taking values in the ideal $\mathfrak{I}$, constructive descriptions of monogenic functions taking values in the mentioned algebras by means of holomorphic functions of the complex variable are obtained in the paper [11].

Let us emphasize that operators generalized inverse to the operators $A_{k}$ can not be expressed in the form of principal extensions of holomorphic functions of the complex variable. Indeed, in the general case, the mentioned principal extensions are not defined in the domain $\Omega$ where a monogenic function $\Phi$ : $\Omega \rightarrow \mathbb{A}_{n}$ is given.

In what follows, $\xi_{k}:=f_{k}(\zeta)$ for all $\zeta \in E_{m}$.

Let us introduce the linear operator $B_{k}$ which assigns a function $\Phi_{k}: \Omega \rightarrow$ $\mathbb{A}_{n}$ to every holomorphic function $F_{k}: D_{k} \rightarrow \mathbb{C}$ by the following formula:

$$
\Phi_{k}(\zeta)=F_{k}\left(\xi_{k}\right) I_{k} \quad \forall \zeta \in \Omega .
$$

Lemma 2. Let a domain $\Omega \subset E_{m}$ be convex with respect to the set of directions $M_{k}$ for some $k \in\{1,2, \ldots, n\}, f_{k}\left(E_{m}\right)=\mathbb{C}$ and a function $F_{k}: D_{k} \rightarrow \mathbb{C}$ be holomorphic in the domain $D_{k}$. Then the function (9) is monogenic in $\Omega$, and the Gateaux s-th derivative $\Phi_{k}^{(s)}$ is a monogenic function in $\Omega$ for any $s$.

Proof. Let $h:=\sum_{j=1}^{m} h_{j} e_{j} \in E_{m}$ be an arbitrary nonzero element. Denote $\eta_{k}:=f_{k}(h)=h_{1}+\sum_{j=2}^{m} a_{j k} h_{j}$, where $a_{j k}$ are the coefficients of decomposition (5). It is follows from the equalities (3) and (5) that $\eta_{k} I_{k}=h I_{k}$. 
Therefore, in the case where $\eta_{k} \neq 0$ we have the following relations:

$$
\begin{gathered}
\lim _{\varepsilon \rightarrow 0+0} \frac{\Phi_{k}(\zeta+\varepsilon h)-\Phi_{k}(\zeta)}{\varepsilon}=I_{k} \lim _{\varepsilon \rightarrow 0+0} \frac{F_{k}\left(\xi_{k}+\varepsilon \eta_{k}\right)-F_{k}\left(\xi_{k}\right)}{\varepsilon}= \\
=\eta_{k} I_{k} \lim _{\varepsilon \rightarrow 0+0} \frac{F_{k}\left(\xi_{k}+\varepsilon \eta_{k}\right)-F_{k}\left(\xi_{k}\right)}{\varepsilon \eta_{k}}=h I_{k} F_{k}^{\prime}\left(\xi_{k}\right) .
\end{gathered}
$$

If $\eta_{k}=0$, then $h \in \mathfrak{I}_{k}$. Therefore, $h I_{k}=0$ and the following equalities hold:

$$
\lim _{\varepsilon \rightarrow 0+0} \frac{\Phi_{k}(\zeta+\varepsilon h)-\Phi_{k}(\zeta)}{\varepsilon}=I_{k} \lim _{\varepsilon \rightarrow 0+0} \frac{F_{k}\left(\xi_{k}\right)-F_{k}\left(\xi_{k}\right)}{\varepsilon}=0 .
$$

Thus, the function (9) is monogenic in $\Omega$ and $\Phi_{k}^{\prime}(\zeta)=F_{k}^{\prime}\left(\xi_{k}\right) I_{k}$.

In a similar way one can establish that the Gateaux $s$-th derivative $\Phi_{k}^{(s)}$ is a monogenic function in $\Omega$ for any $s$. Lemma 2 is proved.

It is clear that $B_{k}$ is a generalized inverse operator for the operator $A_{k}$, i.e. $A_{k} B_{k} A_{k}=A_{k}$.

Lemma 3. Let a domain $\Omega \subset E_{m}$ be convex with respect to the set of directions $M_{k}$ for some $k \in\{1,2, \ldots, n\}$ and $f_{k}\left(E_{m}\right)=\mathbb{C}$. Then every monogenic function $\Phi: \Omega \rightarrow \mathbb{A}_{n}$ can be expressed in the form

$$
\Phi(\zeta)=B_{k} A_{k} \Phi(\zeta)+\Phi_{0 k}(\zeta) \quad \forall \zeta \in \Omega,
$$

where $\Phi_{0 k}: \Omega \rightarrow \mathfrak{I}_{k}$ is a monogenic function taking values in the ideal $\mathfrak{I}_{k}$.

Proof. Consider the function $\Phi_{0 k}(\zeta)=\Phi(\zeta)-B_{k} A_{k} \Phi(\zeta)$ which is monogenic in $\Omega$ due to Lemma 2. It is evident that

$$
F_{k}:=A_{k} \Phi_{0 k}=A_{k} \Phi-A_{k} B_{k} A_{k} \Phi=A_{k} \Phi-A_{k} \Phi=0 .
$$

Therefore, taking into account the equality (8), we obtain

$$
F_{k}\left(\xi_{k}\right)=f_{k}\left(\Phi_{0 k}(\zeta)\right)=0 .
$$

Thus, $\Phi_{0 k}(\zeta) \in \mathfrak{I}_{k}$. Lemma 3 is proved.

Associate with a set $\Lambda \subset E_{m}$ the set $\Lambda_{\mathbb{R}}:=\left\{\left(x_{1}, x_{2}, \ldots, x_{m}\right) \in \mathbb{R}^{m}: \zeta=\right.$ $\left.\sum_{j=1}^{m} x_{j} e_{j} \in \Lambda\right\}$ in $\mathbb{R}^{m}$.

In the following lemma we describe all monogenic functions given in a domain $\Omega \subset E_{m}$ and taking values in the ideal $\mathfrak{I}_{k}$.

Lemma 4. If a domain $\Omega \subset E_{m}$ is convex with respect to the set of directions $M_{k}$ and $f_{k}\left(E_{m}\right)=\mathbb{C}$ for all $k \in\{1,2, \ldots, n\}$, then every monogenic function $\Phi_{0 k}: \Omega \rightarrow \mathfrak{I}_{k}$ can be expressed in the form

$$
\Phi_{0 k}(\zeta)=\sum_{p=1, p \neq k}^{n} F_{p}\left(\xi_{p}\right) I_{p} \quad \forall \zeta \in \Omega,
$$


where $F_{p}: D_{p} \rightarrow \mathbb{C}$ is a function holomorphic in the domain $D_{p}$.

Proof. Inasmuch as the function $\Phi_{0 k}$ takes values in the ideal $\mathfrak{I}_{k}$,

$$
\Phi_{0 k}(\zeta)=\sum_{p=1, p \neq k}^{n} W_{p}\left(x_{1}, x_{2}, \ldots, x_{m}\right) I_{p},
$$

where $W_{p}: \Omega_{\mathbb{R}} \rightarrow \mathbb{C}$.

Taking into account the definition of operator $A_{p}$ and the equalities (6), $(10)$, we obtain $F_{p}\left(\xi_{p}\right):=\left(A_{p} \Phi_{0 k}\right)\left(\xi_{p}\right)=f_{p}\left(\Phi_{0 k}(\zeta)\right)=W_{p}\left(x_{1}, x_{2}, \ldots, x_{m}\right)$. Lemma 4 is proved.

The next theorem follows immediately from Lemmas 3,4 .

Theorem 1. Suppose that a domain $\Omega \subset E_{m}$ is convex with respect to the set of directions $M_{k}$ and $f_{k}\left(E_{m}\right)=\mathbb{C}$ for all $k \in\{1,2, \ldots, n\}$. Then every monogenic function $\Phi: \Omega \rightarrow \mathbb{A}_{n}$ can be expressed in the form

$$
\Phi(\zeta)=\sum_{k=1}^{n} F_{k}\left(\xi_{k}\right) I_{k} \quad \forall \zeta \in \Omega
$$

where $F_{k}: D_{k} \rightarrow \mathbb{C}$ is a function holomorphic in the domain $D_{k}$.

Remark. The condition of convexity of $\Omega$ with respect to the set of directions $M_{k}$ is essential for the truth of Theorem 1 in the case $m<2 n$, see Example 1 in [13]. In the case $m=2 n$, the mention condition can be omitted. In this case, the statement of Theorem 1 can be proved in such a way as the Ringleb theorem [3, p. 136] for analytic functions of a bicomplex variable.

It is evident that the next statement follows from the equality (11) because its right-hand part is a monogenic function in the domain $D:=\left\{\zeta \in E_{m}\right.$ : $\left.f_{k}(\zeta) \in D_{k}, k=1,2, \ldots, n\right\}$.

Theorem 2. Suppose that a domain $\Omega \subset E_{m}$ is convex with respect to the set of directions $M_{k}$ and $f_{k}\left(E_{m}\right)=\mathbb{C}$ for all $k \in\{1,2, \ldots, n\}$. Suppose also that a function $\Phi: \Omega \rightarrow \mathbb{A}_{n}$ is monogenic in $\Omega$. Then $\Phi$ can be continued to a function monogenic in the domain $D$.

A. K. Bakhtin [7] proved a polycylindrical Riemann theorem. In particular, it follows from this theorem in the case $E_{m}=\mathbb{A}_{n}$ that one can map the domain $D$ onto the unit polydisk by means a mapping of the form (11) in the domain $\Omega=D$. It is clear that this mapping is a monogenic function in $D$.

The following statement is true for monogenic functions in an arbitrary domain $\Omega$.

Theorem 3. Let $f_{k}\left(E_{m}\right)=\mathbb{C}$ for all $k \in\{1,2, \ldots, n\}$. Then for every monogenic function $\Phi: \Omega \rightarrow \mathbb{A}_{n}$ in an arbitrary domain $\Omega \subset E_{m}$, the Gateaux $s$-th derivative $\Phi^{(s)}$ is a monogenic function in $\Omega$ for any $s$. 
Proof. Inasmuch as a ball is a convex set, in a ball $\Theta \subset \Omega$ with the center in an arbitrary point $\zeta_{0} \in \Omega$ we have the equality (11). Now, the statement of theorem follows from Lemma 2 . The theorem is proved.

2. Integral theorem for a curvilinear integral.

In the paper [15] for functions differentiable in the sense of Lorch in an arbitrary convex domain of a commutative associative Banach algebra, some properties similar to properties of holomorphic functions of complex variable (in particular, the integral Cauchy theorem and the integral Cauchy formula and the Morera theorem) are established. The convexity of the domain in the mentioned results from [15] is withdrawn by E. K. Blum [16].

In this paper, using the representation (11) of monogenic functions, we prove the integral Cauchy theorem and the integral Cauchy formula for monogenic functions $\Phi: \Omega \rightarrow \mathbb{A}_{n}$ given only in a domain $\Omega$ of the linear span $E_{m}$ instead of domain of whole algebra $\mathbb{A}_{n}$.

Let us note that a priori the differentiability of the function $\Phi$ in the sense of Gateaux is a restriction weaker than the differentiability of this function in the sense of Lorch. Moreover, note that the integral Cauchy formula established in the papers $[15,16]$ is not applicable to a monogenic function $\Phi: \Omega \rightarrow \mathbb{A}_{n}$ because it deals with an integration along a curve on which the function $\Phi$ is not given, generally speaking.

We say that $\gamma$ is a Jordan rectifiable curve in $E_{m}$ if $\gamma_{\mathbb{R}}$ is a Jordan rectifiable curve in $\mathbb{R}^{m}$.

For a continuous function $\Psi: \gamma \rightarrow \mathbb{A}_{n}$ of the form

$$
\Psi(\zeta)=\sum_{k=1}^{n} U_{k}\left(x_{1}, x_{2}, \ldots, x_{m}\right) I_{k}+i \sum_{k=1}^{n} V_{k}\left(x_{1}, x_{2}, \ldots, x_{m}\right) I_{k},
$$

where $\left(x_{1}, x_{2}, \ldots, x_{m}\right) \in \gamma_{\mathbb{R}}$ and $U_{k}: \gamma_{\mathbb{R}} \rightarrow \mathbb{R}, V_{k}: \gamma_{\mathbb{R}} \rightarrow \mathbb{R}$, we define an integral along a Jordan rectifiable curve $\gamma$ by the equality

$$
\begin{gathered}
\int_{\gamma} \Psi(\zeta) d \zeta:=\sum_{j=1}^{m} e_{j} \sum_{k=1}^{n} I_{k} \int_{\gamma_{\mathbb{R}}} U_{k}\left(x_{1}, x_{2}, \ldots, x_{m}\right) d x_{j}+ \\
+i \sum_{j=1}^{m} e_{j} \sum_{k=1}^{n} I_{k} \int_{\gamma_{\mathbb{R}}} V_{k}\left(x_{1}, x_{2}, \ldots, x_{m}\right) d x_{j}
\end{gathered}
$$

where $d \zeta:=e_{1} d x_{1}+e_{2} d x_{2}+\cdots+e_{m} d x_{m}$.

To establish a Cauchy integral theorem for a curvilinear integral, consider the following auxiliary statement:

Lemma 6. Suppose that a domain $\Omega \subset E_{m}$ is convex with respect to the set of directions $M_{k}$ and $f_{k}\left(E_{m}\right)=\mathbb{C}$ for all $k \in\{1,2, \ldots, n\}$. Suppose 
also that $\Phi: \Omega \rightarrow \mathbb{A}_{n}$ is a monogenic function and $\gamma$ is an arbitrary rectifiable curve in $\Omega$. Then

$$
\int_{\gamma} \Phi(\zeta) d \zeta=\sum_{k=1}^{n} I_{k} \int_{\gamma_{k}} F_{k}\left(\xi_{k}\right) d \xi_{k},
$$

where $\gamma_{k}$ is the image of $\gamma$ under the mapping $f_{k}$ and $F_{k}$ is the same function as in (11).

Proof. The equality (13) follows immediately from the representation (11), the equality $d \zeta=d \xi_{1} I_{1}+d \xi_{2} I_{2}+\cdots+d \xi_{n} I_{n}$ and the multiplication rules (3). Lemma 6 is proved.

We understand a triangle $\triangle$ as a plane figure bounded by three line segments connecting three its vertices. Denote by $\partial \triangle$ the boundary of triangle $\triangle$ in relative topology of its plane.

Let $\Omega$ be a domain in $E_{m}$ and $\Phi: \Omega \rightarrow \mathbb{A}_{n}$ be a monogenic function in $\Omega$. Inasmuch as every triangle $\triangle \subset \Omega$ can be included in a convex subset of the domain $\Omega$, using Lemma 6 and the integral Cauchy theorem for holomorphic function $F_{k}$, we obtain immediately the following equality:

$$
\int_{\partial \triangle} \Phi(\zeta) d \zeta=0
$$

Now, similarly to the proof of Theorem 3.2 [16] we can prove the following

Theorem 4. Let $\Phi: \Omega \rightarrow \mathbb{A}_{n}$ be a monogenic function in a domain $\Omega \subset E_{m}$. Then for every closed Jordan rectifiable curve $\gamma$ homotopic to a point in $\Omega$, the following equality holds:

$$
\int_{\gamma} \Phi(\zeta) d \zeta=0 .
$$

For functions taking values in the algebra $\mathbb{A}_{n}$, the following Morera theorem can be established in the usual way:

Theorem 5. If a function $\Phi: \Omega \rightarrow \mathbb{A}_{n}$ is continuous in a domain $\Omega \subset E_{m}$ and satisfies the equality (14) for every triangle $\triangle \subset \Omega$, then the function $\Phi$ is monogenic in the domain $\Omega$.

Let $\zeta \in E_{m}$. An inverse element $\zeta^{-1}$ is of the following form:

$$
\zeta^{-1}=\frac{1}{\xi_{1}} I_{1}+\frac{1}{\xi_{2}} I_{2}+\cdots+\frac{1}{\xi_{n}} I_{n}
$$

and it exists if and only if $\xi_{k} \neq 0$ for all $k \in\{1,2, \ldots, n\}$. 
Let $\zeta_{0}=\sum_{k=1}^{n} \xi_{0 k} I_{k}$ (here $\xi_{0 k} \in \mathbb{R}$ ) be a point in a domain $\Omega \subset E_{m}$. In a neighbourhood of $\zeta_{0}$ contained in $\Omega$ let us take a circle $C\left(\zeta_{0}\right)$ with the center at the point $\zeta_{0}$. By $C_{k}$ we denote the image of $C\left(\zeta_{0}\right)$ under the mapping $f_{k}$. We assume that the circle $C\left(\zeta_{0}\right)$ embraces the set $\left\{\zeta_{0}+\zeta: \zeta \in \bigcup_{k=1}^{n} M_{k}\right\}$. It means that $C_{k}$ bounds a domain $D_{k}^{\prime}$ and $\xi_{0 k} \in D_{k}^{\prime}$ for all $k \in\{1,2, \ldots, n\}$.

We say that the curve $\gamma \subset \Omega$ embraces once the set $\left\{\zeta_{0}+\zeta: \zeta \in \bigcup_{k=1}^{n} M_{k}\right\}$, if there exists a circle $C\left(\zeta_{0}\right)$ which embraces the mentioned set and is homotopic to $\gamma$ in the domain $\Omega \backslash\left\{\zeta_{0}+\zeta: \zeta \in \bigcup_{k=1}^{n} M_{k}\right\}$.

Theorem 6. Suppose that a domain $\Omega \subset E_{m}$ is convex with respect to the set of directions $M_{k}$ and $f_{k}\left(E_{m}\right)=\mathbb{C}$ for all $k \in\{1,2, \ldots, n\}$. Suppose also that $\Phi: \Omega \rightarrow \mathbb{A}_{n}$ is a monogenic function in $\Omega$. Then for every point $\zeta_{0} \in \Omega$ the following equality is true:

$$
\Phi\left(\zeta_{0}\right)=\frac{1}{2 \pi i} \int_{\gamma} \Phi(\zeta)\left(\zeta-\zeta_{0}\right)^{-1} d \zeta
$$

where $\gamma$ is an arbitrary closed Jordan rectifiable curve in $\Omega$, that embraces once the set $\left\{\zeta_{0}+\zeta: \zeta \in \bigcup_{k=1}^{n} M_{k}\right\}$.

Proof. Inasmuch as $\gamma$ is homotopic to $C\left(\zeta_{0}\right)$ in the domain $\Omega \backslash\left\{\zeta_{0}+\zeta\right.$ : $\left.\zeta \in \bigcup_{k=1}^{n} M_{k}\right\}$, it follows from Theorem 4 that

$$
\frac{1}{2 \pi i} \int_{\gamma} \Phi(\zeta)\left(\zeta-\zeta_{0}\right)^{-1} d \zeta=\frac{1}{2 \pi i} \int_{C\left(\zeta_{0}\right)} \Phi(\zeta)\left(\zeta-\zeta_{0}\right)^{-1} d \zeta .
$$

Further, using the equality (15), Lemma 6 and the integral Cauchy formula for holomorphic function $F_{k}$, we obtain immediately the following equalities:

$$
\begin{gathered}
\frac{1}{2 \pi i} \int_{C\left(\zeta_{0}\right)} \Phi(\zeta)\left(\zeta-\zeta_{0}\right)^{-1} d \zeta=\sum_{k=1}^{n} I_{k} \frac{1}{2 \pi i} \int_{C_{k}} \frac{F_{k}\left(\xi_{k}\right)}{\xi_{k}-\xi_{0 k}} d \xi_{k}= \\
=\sum_{j=k}^{n} F_{k}\left(\xi_{0 k}\right) I_{k}=\Phi\left(\zeta_{0}\right),
\end{gathered}
$$

where $\zeta_{0}=\xi_{01} I_{1}+\xi_{02} I_{2}+\cdots+\xi_{0 n} I_{n}$. The theorem is proved.

\section{Examples.}

- Consider the algebra $\mathbb{A}_{2}$. It coincides with the algebra of bicomplex numbers and is isomorphic to the C. Segre [1] algebra of commutative quaternions mentioned in Introduction. It is clear that the basis $e_{1}=1$, $e_{2}=i, e_{3}=j, e_{4}=k$ satisfies the condition

$$
e_{1}^{2}+e_{2}^{2}+e_{3}^{2}+e_{4}^{2}=0,
$$


and monogenic functions $\Phi(\zeta)$ of the variable $\zeta=x e_{1}+y e_{2}+z e_{3}+t e_{4}$ with $x, y, z, t \in \mathbb{R}$ satisfy the four-dimensional Laplace equation because

$$
\frac{\partial^{2} \Phi}{\partial x^{2}}+\frac{\partial^{2} \Phi}{\partial y^{2}}+\frac{\partial^{2} \Phi}{\partial z^{2}}+\frac{\partial^{2} \Phi}{\partial t^{2}}=\Phi^{\prime \prime}(\zeta)\left(e_{1}^{2}+e_{2}^{2}+e_{3}^{2}+e_{4}^{2}\right)=0 .
$$

- Consider in the algebra $\mathbb{A}_{2}$ three elements

$$
e_{1}=1, \quad e_{2}=\frac{i}{\sqrt{2}}, \quad e_{3}=\frac{i}{\sqrt{2}}\left(I_{1}-I_{2}\right),
$$

which satisfy the conditions (1). Then every monogenic function $\Phi(\zeta)$ of the variable $\zeta=x e_{1}+y e_{2}+z e_{3}$ satisfies the three-dimensional Laplace equation due to the equalities (2).

- In the algebra $\mathbb{A}_{3}$, all bases satisfying the equality (1) and the inequality $e_{k}^{2} \neq 0$ for $k=1,2,3$ are described in Theorem 1.10 [10].

- Consider the following basis in $\mathbb{A}_{n}$ :

$$
e_{1}=1, \quad e_{k}=i I_{k-1} \quad \text { for } k=2,3, \ldots, n-1, \quad e_{n}=i I_{n-1}-i I_{n},
$$

that satisfies the equality

$$
e_{1}^{2}+e_{2}^{2}+\cdots+e_{n}^{2}=0 .
$$

Then monogenic functions $\Phi(\zeta)$ of the variable $\zeta=\sum_{k=1}^{n} x_{k} e_{k}$ with $x_{k} \in \mathbb{R}$ satisfy the $n$-dimensional Laplace equation because

$$
\frac{\partial^{2} \Phi}{\partial x_{1}^{2}}+\frac{\partial^{2} \Phi}{\partial x_{2}^{2}}+\cdots+\frac{\partial^{2} \Phi}{\partial x_{n}^{2}}=\Phi^{\prime \prime}(\zeta)\left(e_{1}^{2}+e_{2}^{2}+\cdots+e_{n}^{2}\right)=0 .
$$

- If in the algebra $\mathbb{A}_{n}$ one consider the elements

$$
\begin{gathered}
e_{1}=1, \quad e_{2}=I_{2}, \quad e_{3}=I_{3}, \ldots, \quad e_{n}=I_{n}, \\
e_{n+1}=i, \quad e_{n+2}=i I_{2}, \quad e_{n+3}=i I_{3}, \ldots, \quad e_{2 n}=i I_{n}
\end{gathered}
$$

satisfying the equality

$$
e_{1}^{2}+e_{2}^{2}+\cdots+e_{2 n}^{2}=0,
$$

then monogenic functions $\Phi(\zeta)$ of the variable $\zeta=\sum_{j=1}^{2 n} x_{j} e_{j}$ with $x_{j} \in \mathbb{R}$ satisfy $2 n$-dimensional Laplace equation because

$$
\frac{\partial^{2} \Phi}{\partial x_{1}^{2}}+\frac{\partial^{2} \Phi}{\partial x_{2}^{2}}+\cdots+\frac{\partial^{2} \Phi}{\partial x_{2 n}^{2}}=\Phi^{\prime \prime}(\zeta)\left(e_{1}^{2}+e_{2}^{2}+\cdots+e_{2 n}^{2}\right)=0 .
$$


4. Cauchy integral theorem for a surface integral. Along with monogenic functions $\Phi: \Omega \rightarrow \mathbb{A}_{n}$ satisfying the Cauchy - Riemann conditions (4), consider a hyperholomorphic function $\Psi: \Omega \rightarrow \mathbb{A}_{n}$ having continuous partial derivatives of the first order in a domain $\Omega$ and satisfying the equation

$$
\frac{\partial \Psi}{\partial x_{1}} e_{1}+\frac{\partial \Psi}{\partial x_{2}} e_{2}+\cdots+\frac{\partial \Psi}{\partial x_{m}} e_{m}=0
$$

in every point of this domain.

In the scientific literature the different denominations are used for functions satisfying equations of the form (18). For example, in the papers [17, 18] they are called regular functions, and in the papers $[19,20]$ they are called monogenic functions. We use the terminology of the papers [21, 22].

Note that the class of hyperholomorphic functions does not coincide with the class of monogenic functions. In particular, every monogenic function $\Phi: \Omega \rightarrow \mathbb{A}_{n}$ satisfies the equality (18) due to the equalities (4) in the case where the vectors $e_{1}, e_{2}, \ldots, e_{m}$ satisfy the condition (17), where one ought to set $n=m$.

Let $\Omega$ be a bounded domain in $E_{m}$. For a continuous function $\Psi: \Omega \rightarrow \mathbb{A}_{n}$ of the form (12), where $\left(x_{1}, x_{2}, \ldots, x_{m}\right) \in \Omega_{\mathbb{R}}$ and $U_{k}: \Omega_{\mathbb{R}} \rightarrow \mathbb{R}, V_{k}: \Omega_{\mathbb{R}} \rightarrow \mathbb{R}$, we define a volume integral by the equality

$$
\begin{gathered}
\int_{\Omega} \Psi(\zeta) d x_{1} d x_{2} \ldots d x_{m}:=\sum_{k=1}^{n} I_{k} \int_{\Omega_{\mathbb{R}}} U_{k}\left(x_{1}, x_{2}, \ldots, x_{m}\right) d x_{1} d x_{2} \ldots d x_{m}+ \\
+i \sum_{k=1}^{n} I_{k} \int_{\Omega_{\mathbb{R}}} V_{k}\left(x_{1}, x_{2}, \ldots, x_{m}\right) d x_{1} d x_{2} \ldots d x_{m} .
\end{gathered}
$$

We say that $\Sigma$ is a piece-smooth hypersurface in $E_{m}$ if $\Sigma_{\mathbb{R}}$ is a piecesmooth hypersurface in $\mathbb{R}^{m}$. For a continuous function $\Psi: \Sigma \rightarrow \mathbb{A}_{n}$ of the form (12), where $\left(x_{1}, x_{2}, \ldots, x_{m}\right) \in \Sigma_{\mathbb{R}}$ and $U_{k}: \Sigma_{\mathbb{R}} \rightarrow \mathbb{R}, V_{k}: \Sigma_{\mathbb{R}} \rightarrow \mathbb{R}$, we define a surface integral on a piece-smooth surface $\Sigma$ with the differential form $\sigma:=\sum_{j=1}^{m} e_{j} \bigwedge_{q=1, q \neq j}^{m} d x_{q}$ by the equality

$$
\begin{gathered}
\int_{\Sigma} \Psi(\zeta) \sigma:=\sum_{j=1}^{m} e_{j} \sum_{k=1}^{n} I_{k} \int_{\Sigma_{\mathbb{R}}} U_{k}\left(x_{1}, x_{2}, \ldots, x_{m}\right) \bigwedge_{q=1, q \neq j}^{m} d x_{q}+ \\
+i \sum_{j=1}^{m} e_{j} \sum_{k=1}^{n} I_{k} \int_{\Sigma_{\mathbb{R}}} V_{k}\left(x_{1}, x_{2}, \ldots, x_{m}\right) \bigwedge_{q=1, q \neq j}^{m} d x_{q} .
\end{gathered}
$$


If a domain $\Omega \subset E_{m}$ has a closed piece-smooth boundary $\partial \Omega$ and a function $\Psi: \Omega \rightarrow \mathbb{A}_{n}$ is continuous together with partial derivatives of the first order up to the boundary $\partial \Omega$, then the following analogue of the Gauss - Ostrogradsky formula is true:

$$
\int_{\partial \Omega} \Psi(\zeta) \sigma=\int_{\Omega}\left(\sum_{j=1}^{m} \frac{\partial \Psi}{\partial x_{j}} e_{j}\right) d x_{1} d x_{2} \ldots d x_{m} .
$$

Now, the next theorem is a result of the formula (19) and the equality (18).

Theorem 7. Suppose that $\Omega$ has a closed piece-smooth boundary $\partial \Omega$. Suppose also that the function $\Psi: \Omega \rightarrow \mathbb{A}_{n}$ is hyperholomorphic in $\Omega$ and is continuous together with partial derivatives of the first order up to the boundary $\partial \Omega$. Then

$$
\int_{\partial \Omega} \Psi(\zeta) \sigma=0 .
$$

Note that an analogue of the Cauchy integral theorem for a surface integral is proved in the paper [23] for hyperholomorphic functions given in domains with non piece-smooth boundaries and taking values in an arbitrary finitedimensional commutative associative Banach algebra but in the case of threedimensional domains only.

Acknowledgements. The publication of this paper is partially supported by the grant PN-II-ID- WE-2012-4-169.

\section{References}

[1] C. Segre, The real representations of complex elements and extentions to bicomlex systems, Math. Ann., 40(1892), 413 - 467.

[2] F. Ringleb, Beiträge zur funktionentheorie in hyperkomplexen systemen, I, Rend. Circ. Mat. Palermo, 57(1)(1933), $311-340$.

[3] J.D. Riley, Contributions to the theory of functions of a bicomplex variable, Tohoku Math. J., 5(2)(1953), 132 - 165.

[4] G. B. Price, An introduction to multicomplex spaces and functions, New York, Marcel Dekker, 1991.

[5] S. Rönn, Bicomplex algebra and function theory, arXiv: math/0101200v1 [math.CV], 24 Jan 2001. 
[6] D. A. Pinotsis, Commutative quaternions, spectral analysis and boundary value problems, Complex Variables and Elliptic Equations, 57(9)(2012), $953-966$.

[7] A.K. Bakhtin, A generalization of some results in the theory of univalent functions on a multi-dimensional complex space, Dop. NAN Ukr., (3)(2011), 7 - 11 [in Russian].

[8] P.W. Ketchum, Analytic functions of hypercomplex variables, Trans. Amer. Math. Soc., 30(4)(1928), $641-667$.

[9] I. P. Mel'nichenko, The representation of harmonic mappings by monogenic functions, Ukr. Math. J., 27(5)(1975), $499-505$.

[10] I. P. Mel'nichenko and S. A. Plaksa, Commutative algebras and spatial potential fields, Kiev, Inst. Math. NAS Ukraine, 2008 [in Russian].

[11] S. A. Plaksa, S. V. Grishchuk and V.S. Shpakivskyi, Commutative algebras of monogenic functions associated with classic equations of mathematical physics, Contemporary Mathematics, 553(2011), 245 - 258.

[12] S. A. Plaksa and R. P. Pukhtaievych, Constructive description of monogenic functions in a three-dimensional harmonic algebra with onedimensional radical, Ukr. Math. J., 65(5) (2013), 670 - 680.

[13] R.P. Pukhtaievych, Monogenic functions in a three-dimensional harmonic semi-simple algebra, Zb. Pr. Inst. Mat. NAN Ukr., 10(4-5)(2013), $352-361$.

[14] S. A. Plaksa, Commutative algebras associated with classic equations of mathematical physics, Advances in Applied Analysis, Trends in Mathematics, Springer, Basel, 2012, $177-223$.

[15] E. R. Lorch, The theory of analytic runction in normed abelin vector rings, Trans. Amer. Math. Soc., 54(1943), $414-425$.

[16] E.K. Blum, A theory of analytic functions in banach algebras, Trans. Amer. Math. Soc., 78(1955), $343-370$.

[17] A. Sudbery, Quaternionic analysis, Math. Proc. Camb. Phil. Soc., 85(1979), $199-225$.

[18] W. Sprößig, Eigenvalue problems in the framework of Clifford analysis, Advances in Applied Clifford Algebras, 11(2001), 301 - 316. 
[19] F. Brackx and R. Delanghe, Duality in hypercomplex functions theory, J. Funct. Anal., 37(2)(1980), 164-181.

[20] S. Bernstein, Factorization of the nonlinear Schrödinger equation and applications, Complex Variables and Elliptic Equations, 51(5-6)(2006), 429-452.

[21] V. V. Kravchenko and M. V. Shapiro, Integral representations for spatial models of mathematical physics, Pitman Research Notes in Mathematics, Addison Wesley Longman Inc, 1996.

[22] B. Schneider and E. Karapinar, A note on biquaternionic MIT bag model, Int. J. Contemp. Math. Sci., 1(10)(2006), 449-461.

[23] S. A. Plaksa and V.S. Shpakivskyi, Cauchy theorem for a surface integral in commutative algebras, Complex Variables and Elliptic Equations, $\mathbf{5 9}(1)(2014), 110-119$.

Sergey PLAKSA,

Department of complex analysis and potential theory,

Institute of Mathematics of the National Academy of Sciences of Ukraine,

3 Tereshchenkivska Street, Kiev, Ukraine

Email: plaksa@imath.kiev.ua

Roman PUKHTAIEVYCH,

Department of complex analysis and potential theory,

Institute of Mathematics of the National Academy of Sciences of Ukraine,

3 Tereshchenkivska Street, Kiev, Ukraine

Email: p_r_p@imath.kiev.ua 